\title{
Characterization of non-tuberculous mycobacterium from humans and water in an Agropastoral area in Zambia
}

\author{
Ngula Monde ${ }^{1,2^{*}}$, Musso Munyeme ${ }^{2}$, Adrian Muwonge ${ }^{3}$, John Bwalya Muma² and Sydney Malama ${ }^{4}$
}

\begin{abstract}
Background: The non-tuberculous mycobacteria include those mycobacterium species that are not members of the Mycobacterium tuberculosis complex, the causative agent of pulmonary tuberculosis and Mycobacterium leprae. In Zambia, Non-tuberculous Mycobacteria are gaining recognition as pathogens of public health significance. However, there is scanty information on the isolation and speciation of these organisms for better patient management, consequently reducing the burden of these infections. Given the above information, the thrust of this study was to isolate and characterize NTM from humans and water in Namwala district of Zambia.
\end{abstract}

Method: This was a cross-sectional study were 153 individuals with suspected TB were sampled from four health facilities in Namwala district, sputum samples were also collected. Additionally, 149 water samples were collected from different water drinking sources such as Tap water, Borehole water, rivers, wells and streams. Standard TB culture methods were employed to isolate Non-tuberculous Mycobacteria and later 165-23S internal transcribed spacer region Sequencing was employed to characterize NTM.

Results: Seven (7, 4.6\%) NTM species were identified from humans with $M$. arupense $(3,42.9 \%)$ being the most common organism, while twenty three $(23,15.4 \%)$ NTM were identified from water with the common species being Mycobacterium gordonae (5, 21.7\%). Mycobacterium avium and Mycobacterium fortuitum were both identified from human and water samples.

Conclusion: This study has shown the isolation of NTM species from humans and water. The isolation of NTM from drinking water sources could signify a public health risk to humans.

Keywords: Namwala, NTM, Water, Mycobacteria, Zambia

\section{Background}

The Non-tuberculous Mycobacteria (NTM) include those mycobacterium species that are not members of the Mycobacterium tuberculosis complex (MTC), the causative agent of pulmonary tuberculosis (TB) and Mycobacterium laprae [2, 3, 24]. They are normal inhabitants of natural waters, drinking waters, animals, birds and soils [25, 33, 41]. The environment is the primary source of infection of these mycobacteria to humans. NTM have also been recovered from meat, fish, dairy products, fruits, vegetables and

\footnotetext{
*Correspondence: nkabelenga@yahoo.co.uk

${ }^{1}$ Department of Biomedical Sciences, Tropical Diseases Research Center,

P.O.Box 71769, Ndola, Zambia

${ }^{2}$ Department of Disease Control, School of Veterinary Medicine, University of

Zambia, P.O. Box 32379, 10101 Lusaka, Zambia

Full list of author information is available at the end of the article
}

specially unpasteurized milk suggesting a public health risk for these bacteria [19].

Human-to-human transmission of NTM is generally uncommon, although there is evidence of transmission of certain NTM species such as Mycobacterium kansasii [33].

Although members of the MTC are largely known to cause the majority of the mycobacterial infections worldwide, infections due to NTM are also emerging [17]. There are 172 different species of NTM but the most potentially pathogenic of these are Mycobacterium avium, Mycobacterium intracellulare, Mycobacterium kansasii, Mycobacterium xenopi, and Mycobacterium abscessus [17]. These organisms are capable of causing pulmonary disease, disseminated disease and localized lesions, mostly in immune-compromised individuals. Pulmonary diseases 
are the most frequently encountered and have been reported to account for up to $94 \%$ of cases of NTM associated disease [28]. People living with Human Immunodeficiency Virus (HIV) and Acquired Immunodeficiency Syndrome (HIV/ AIDS), represent one of the most vulnerable populations to NTM infections, as well as a higher risk for complications and poor disease outcomes. Those receiving immunosuppressive therapy secondary to organ transplantation, cancer, autoimmune disease and those with diabetes mellitus may also be more susceptible to NTM infections compared to the general population [2].

Non-tuberculous Mycobacteria can display clinical and radiological features similar to those exhibited by MTC, hence the need for species identification. Distinguishing between MTC and NTM infections may be challenging, especially in low income countries such as Zambia where Acid Fast Bacilli (AFB) smear microscopy is mainly used for MTC diagnosis. Even though AFB smear microscopy, allows for rapid diagnosis of mycobacteria, it does not differentiate MTC from NTM. This may lead to NTM related infections being under-diagnosed or misdiagnosed as TB [7].

In Zambia, NTM have also been recognized as pathogens of major public health significance with the advent of HIV and AIDS [5, 6, 21]. However, only scanty studies have demonstrated the isolation and characterization of mycobacteria species in humans and water. This study therefore, aimed at isolation and characterization of NTM from humans and water in Namwala district of Zambia.

\section{Methods}

\section{Study area}

The study was conducted in Namwala district of Zambia. Namwala is a rural district situated in the southern province of Zambia and is one of the districts with a large number of pastoral farmers. It has the highest TB prevalence in both animals and humans [22, 23]. It covers an estimated total area of about $10,000 \mathrm{~km}^{2}$ and lies between latitudes 15 and $17^{\circ} \mathrm{S}$ of the equator and longitude 25 and $27^{\circ}$ E. (Fig. 1). The greater area of its traditional land is covered by the Kafue flood plains of the Kafue River. These offer nutritive varieties of rich lush green grass for both cattle and wildlife for a greater part of the year than the surrounding Savannah woodlands [24]. It supports approximately 30,000 herds of cattle and 44,000 Kafue lechwe [22]. Agriculture is the main economic activity, and majority of the people keep livestock. Humans and animals commonly share the same micro-environments such as water drinking points, especially in dry seasons [30], increasing the possibility of interspecies disease transmission or contamination of water sources, which could potentially lead to a zoonotic transmission to humans [18].

\section{Study design}

This was a cross-sectional study in adolescent and adult presumptive TB patients. In addition, water samples were collected. For human sampling, sputum samples were collected from patients seeking medical attention at four local health centers in Namwala district, namely, Namwala district Hospital, Chitongo Health Centre, Kabulamwanda Health Centre and Maala Rural Health Centre. Patients who presented with TB-like symptoms such as chronic (Defined as $\geq 2$ weeks) productive cough, loss of appetite, fever, fatigue, headache and night sweats were enrolled in the study. Participants who presented with other ailment were not included in our study.

Water samples were collected from common water drinking points in areas where the study participants came from. The water collection points comprised of taps, communal boreholes, open wells, and streams/ river. Community health workers in these areas helped in accessing these water points. Our sampling was purposive as study subjects were selected based on the above mentioned characteristics.

\section{Study period}

The study was carried out between October 2015 and March 2016.

\section{Laboratory methods Sputum samples}

After routine microscopy with the Ziehl-Neelsen (ZN) staining technique, the samples were stored in cetylpyridinium chloride (CPC) transport medium (Sigma-Aldrich, Steinheim, Germany, a mixture of $1.0 \% \mathrm{CPC}$ and $2.0 \% \mathrm{NaCl}$ ) and kept at ambient temperature at the health facilities until they were taken to Tropical Diseases Research Centre (TDRC), TB Reference Laboratory (Ndola) for processing. At TDRC TB Reference Laboratory, samples were decontaminated using the Petroff method [29] and cultured on Lowenstein-Jensen (LJ) medium. The cultures were incubated at $37{ }^{\circ} \mathrm{C}$ and examined for growth weekly for at least 8 weeks and results recorded. Microscopic examination of positive cultures using $\mathrm{ZN}$ staining method was performed to detect the presence of AFB.

\section{Water samples}

One hundred and forty nine (149) water samples were collected from various drinking points such as streams, boreholes, tap and rivers in Namwala Central, Maala, Kabulamwanda and Chitongo. An amount of $150 \mathrm{ml}$ water was collected in sterile whirlpak plastic bags and immediately placed in a cool box containing ice packs and transported to the laboratory where they were stored in CPC media (BD Diagnostics, Sparks, MD) until processed. 


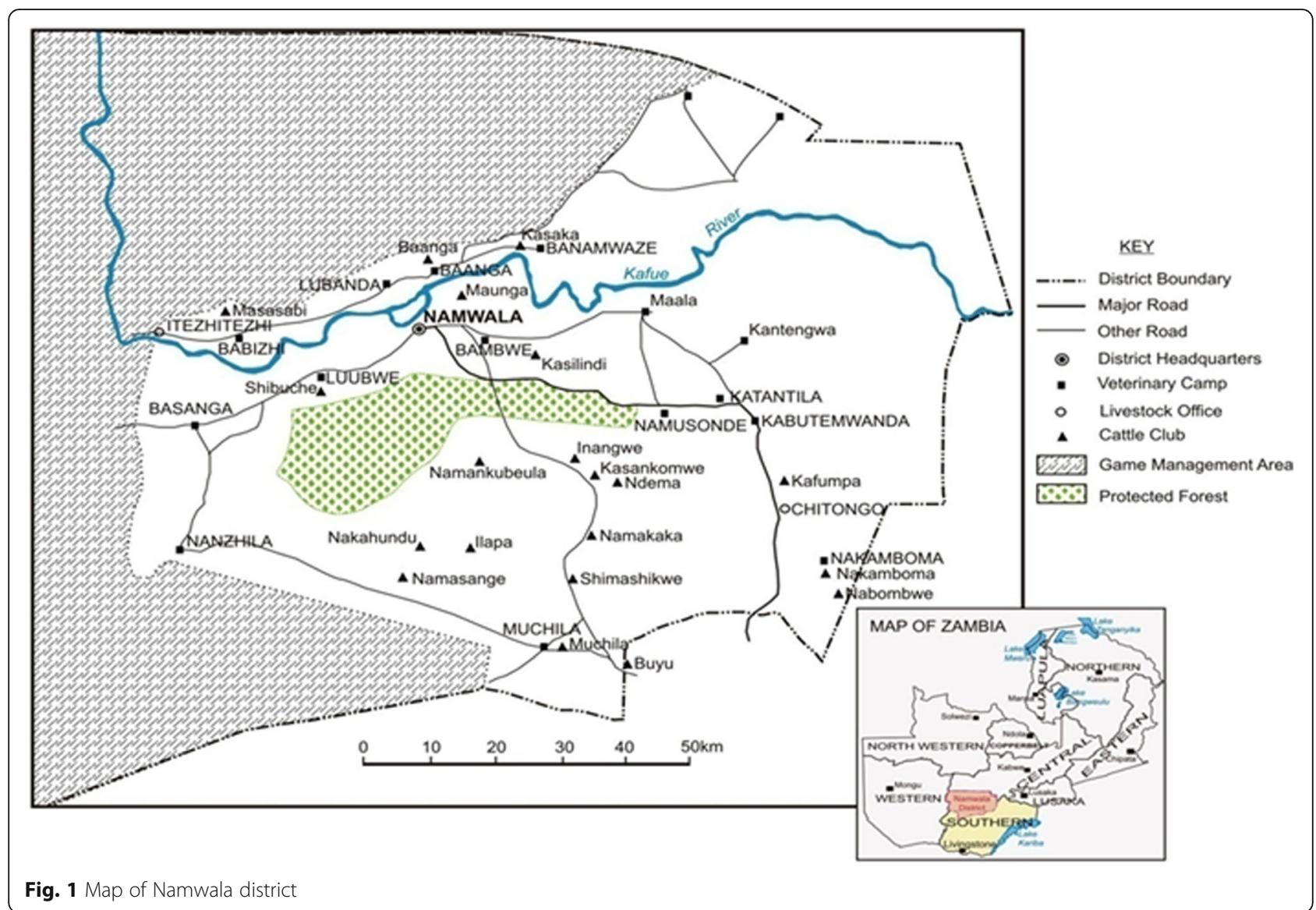

Hundred and Fifty $(150 \mathrm{ml})$ of water was filtered through $0.45 \mu \mathrm{m}$ nitrocellulose membrane filters (Millipore Corporation, Bedford, MS, USA) by vacuum filtration using a Manifold Filtration System (Sartorius AG, Goettingen, Germany). The membranes were transferred into sterile screw-capped specimen containers with $10 \mathrm{ml}$ of $0.005 \%$ $\mathrm{CPC}$. The surface of the membranes was abraded vigorously and thoroughly with a sterile plastic inoculating loop. The samples were then exposed to $0.005 \%$ CPC for $30 \mathrm{~min}$ at room temperature. The mixture was then centrifuged at $3000 \times$ g for $20 \mathrm{~min}$. The pellet was washed twice with $1 \mathrm{ml}$ sterile normal saline and finally re-suspended in $1 \mathrm{ml}$ sterile distilled water. The suspension $(0.5 \mathrm{ml})$ was inoculated on LJ slants and incubated at $37{ }^{\circ} \mathrm{C}$. The cultures were examined for growth for up to 8 weeks and results recorded. Microscopic examination of positive cultures was done using Ziehl Neelsen staining method to detect the presence of AFB.

\section{Sequencing of 16S- 23S rRNA ITS region}

Upon detection of AFB in positive cultures, the Mycobacterial growth was identified as either MTC or NTM using a rapid identification test known as Capilia TB-Neo Kit (TAUNS Laboratories, Inc. Japan), following manufacturer's instructions (capilia.co.jp/english/capilia_tb-neo.html). To obtain genomic DNA for sequencing, the invitrogen TRIzol $^{\mathrm{TM}}$ Reagent was used for DNA extraction according to Hongbao et al. [15]. Thereafter, polymerase chain reactions (PCRs) were performed followed by sequencing of the $16 \mathrm{~S}$ - 23SrRNA Internal Transcribed Spacer region with primers Sp1 (5'-ACC TCC TTT CTA AGG AGC ACC-3') and Sp2 (5'-GAT GCT CGC AAC CAC TAT CCA-3') [34]. Sequencing was performed using an ABI sequencer (Applied Biosystem Inc.) at the school of Veterinary Medicine, University of Zambia. The obtained sequences were aligned using MEGA version 7and the alignments were then blasted against the NBCI database with Mycobacterium taxid 85,007 as the reference organism. The strain name with the highest E.value and identity hit and score was chosen.

\section{Results}

Isolation of NTM in sputum and water samples

Based on morphological characteristics on LJ media and acid fastness by the ZN method, 17 (11.11\%) mycobacterial species were detected out of the 153 study participants. Seven (7) (4.6\%) were identified as NTM while 10 (6.5\%) were MTC. However, co-infection of NTM and MTC was not observed among the suspected TB patients. 
Thirty two 32 (21.5\%) NTM were isolated from 149 water samples collected. The majority of NTM were isolated from borehole water sources $17(53.1 \%)$ followed by river 9 (28.1\%), stream 3 (9.4\%), dam 2 (6.3\%), and tap $1(3.1 \%)$ water sources.

\section{Molecular characterization of NTM in humans and water} All the seven (7) NTM isolated were characterized to species level, with the most isolated species being Mycobacterium arupense 3 species (42.9\%) (Table 1).

Out of 32 NTM isolated from water samples, 23 NTM species were characterized with the most common being Mycobacterium gordonae, 5 species (21.7\%); Mycobacterium senegalense, 3 species (13.0\%); Mycobacterium peregrinum, 3 species (13.0\%), Mycobacterium fortuitum, 3 species (13.0\%) and Mycobacterium gilvum, 2 species (8.7\%) (Table 1). Nine (9) NTM were uncharacterized. Mycobacterium avium and Mycobacterium fortuitum were both isolated in humans and water samples. Of the four study sites in Namwala district, Maala had the most NTM species identified (11 species) while Chitongo had the least NTM species (2 species).

\section{Discussion}

Non-tuberculous Mycobacteria have gained much clinical significance in the last couple of decades in both immunocompromised and immune-competent individuals [1].

Table 1 NTM species isolated in Humans and Water in Namwala district

\begin{tabular}{lll}
\hline NTM species & $n$ & Frequency (\%) \\
\hline Humans $(N=153, n=7)$ & 3 & 42.9 \\
M. arupense & 1 & 14.3 \\
M. abscesus & 1 & 14.3 \\
M. avium & 1 & 14.3 \\
M. fortuitum & 1 & 14.3 \\
M. nebraskense & & \\
Water (N=149, $n=23)$ & 5 & 21.7 \\
M. gordonae & 5 & 13.0 \\
M. senegalense & 3 & 13.0 \\
M. fortuitum & 3 & 13.0 \\
M. peregrinum & 3 & 4.3 \\
M. kumamotonense & 1 & 4.3 \\
M. simiae & 1 & 4.3 \\
M. avium & 1 & 8.7 \\
M. gilvum & 2 & 4.3 \\
M. flavescens & 1 & 4.3 \\
M. housetonense & 1 & 4.3 \\
M. smegmatis & 1 & 4.3 \\
M. parafinicum & 1 & \\
\hline
\end{tabular}

Water is a documented source of NTM infection in humans, but it is not the only source [38]. Currently, very little data exists on the NTM species distribution in humans and water in Africa, Zambia inclusive. It was against this background that this present study was formulated and to the best of our knowledge, this might be the first study in Zambia to isolate and characterize NTM from humans and water simultaneously.

In the present study, a range of NTM from humans and water in Namwala district, an agro-pastoral district located in Southern province of Zambia were isolated. The study found the overall prevalence of NTM in humans in Namwala district to be $4.6 \%$, among patients presenting with TB-like symptoms at local health centers. This is comparable to a study done in Shanghai China, were the overall prevalence of NTM in humans was $5.9 \%[44]$.

In the current study, out of 149 water samples collected from various study sites in Namwala district, 32 (21.5\%) samples were positive for NTM using phenotypic identification method, indicating that NTM were common in water sources of Namwala district.

Our study also found a high isolation rate of NTM in borehole water sources followed by river, dam, stream and lowest in tap water sources. The most prevalent NTM species isolated was Mycobacterium gordonae. Although another study carried out in Sri Lanka [10] showed a high frequency of isolation of mycobacteria from aquarium water and surface water (rivers, streams) with the most common NTM being Mycobacterium fortuitum), These findings in our study could be due to high levels of organic matter and soil in borehole waters and surface waters (rivers, dams, streams) contributing to the mycobacterial flora. The piping systems used in boreholes may support the formation of biofilms which favors the growth and multiplication of NTM [37]. Some pipe materials have been shown to contribute to biofilm formation particularly iron pipes [37] The capacity of mycobacteria to form biofilms has been demonstrated in studies done elsewhere $[9,13]$. In Germany, $90 \%$ of biofilm samples from pipes of various water distribution systems contained Mycobacteria, signifying that Mycobacteria biofilms are present in piping systems [35]. The low mycobacterial load in tap water sources observed in our study could probably be related to the lethal effect of chlorine to mycobacteria, since tap water is usually chlorinated in Namwala district by the local water supply company.

The isolation rate of NTM in both water and human samples was also highest in Maala area as compared to the other study sites in Namwala district of Zambia. This can be attributed to the fact that Maala is surrounded by open grasslands with a lot of Dambos, rivers and papyrus flora, with reeds that enhance the 
formation of microfilms and subsequently increase the multiplication of NTM [13].

A range of pathogenic NTM species were also isolated in the present study and the most common organism was Mycobacterium arupense. This is the first study in Zambia to report the isolation of Mycobacterium arupense in human sputum samples. Cloud et al., [8] identified Mycobacterium arupense for the first time in human clinical samples in the United States of America. Other studies done in Japan [26], Taiwan [40]; and in Greece [27] also reported the isolation of Mycobacterium arupense in human sputum samples. In all these four studies Mycobacterium arupense was of clinical significance. Mycobacterium arupense is a novel mycobacterium species potentially pathogenic and has been associated with causing human pulmonary infections [36]. Therefore, Mycobacterium arupense isolated from humans in this study could possibly be of clinical significance as the patients presented with symptoms of pulmonary infection.

Other NTM species that were isolated in humans were Mycobacterium abscessus, Mycobacterium avium, Mycobacterium fortuitum and Mycobacterium nebraskense. All these species have been found to cause disease in immune compromised and immune competent individuals. Mycobacterium abscessus has been reported to be the leading cause of lower respiratory tract infections among the rapidly growing NTM species $[6,16]$. Mycobacterium avium has also been known to cause opportunistic infections in humans and animals [11] while Mycobacterium nebraskense is an NTM pathogen that has been associated with nodular pulmonary disease [32].

Drinking water has been suggested as a reservoir for many pathogenic and potentially pathogenic NTM such as Mycobacterium avium complex, Mycobacterium gordonae, Mycobacterium kansasii, Mycobacterium fortuitum, Mycobacterium simiae, Mycobacterium senegalense, Mycobacterium chelonae and Mycobacterium xenopi [12, $31,38]$. In our study, a number of pathogenic and potentially pathogenic NTM species were isolated from water samples of Namwala district. The most prevalent NTM species was Mycobacterium gordonae, followed by Mycobacterium fortuitum, Mycobacterium senegalense, and Mycobacterium peregrinum. This is in partial agreement with the findings of the study conducted by Thomson and others in Brisbane, Australia, where Mycobacterium gordonae was the most commonly isolated species in natural and municipality water sources [38]. Mycobacterium gordonae is one of the least pathogenic of the mycobacterium species isolated. It is usually a contaminant or colonizer in immune competent individuals; however, it has been found to be pathogenic in HIV infected patients who are severely immune compromised [4]. Apart from AIDS, underlying immunue suppression, advanced structural lung disease such as emphysema, pneumoconiosis, alcoholism, chronic lung disease, diabetes mellitus and malignancy have all been reported as risk factors for invasive infections $[4,43]$. Therefore, Mycobacterium gordonae isolated from clinical samples should not be automatically considered as a contaminant or colonizer especially in immune compromised patients or patients with the above mentioned risk factors.

Mycobacterium fortuitum, Mycobacterium senegalense and Mycobacterium peregrinum all belong to the Mycobacterium fortuitum complex. A study done by Velayati et al. [42] also found Mycobacterium fortuitum to be the most common species in water. Immune compromised individuals are more likely to be infected with Mycobacterium fortuitum from water sources. Infections caused by this organism include skin and soft tissue infections, lymphadenitis, pulmonary infections and catheter related infections [11].

Mycobacterium senegalense and Mycobacterium peregrinum have also been isolated in water elsewhere [20,37]. They are potentially pathogenic NTM species and have been implicated to cause disease both in immune competent and immune compromised persons [39]. The isolation of Mycobacterium senegalense in water sources of Namwala district is another key finding in our study. Mycobacterium senegalense is a rapidly growing mycobacterium species that has been reported to cause bovine farcy among cattle in east Africa and bovine farcy has been known to cause huge economic loss [14]. Therefore, isolation of this NTM species in water in the present study poses a risk to cattle in Namwala district because this is a pastoral area [21]. Further, all Mycobacterium senegalense species isolated in this study were from Maala and this area has the largest number of cattle.

\section{Conclusion}

This study has shown the isolation of NTM species from humans and water with Mycobacterium arupense and Mycobacterium gordonae being the most prevalent. However, there is no evidence of human to human acquisition but rather a common environmental source, water. Further, the wide presence of NTM in the water sources of Namwala could pose a risk especially for the immune compromised individuals. Therefore, prevention of public health risk of NTM should take into account water treatment before it's accessed for domestic consumption.

\section{Abbreviations}

AFB: Acid-Fast Bacilli; AIDS: Acquired Immunodeficiency Syndrome; Cl: Confidence Interval; CPC: Cetylpyridinium Chloride;

DNA: Deoxyribonucleic Acid; HIV: Human Immunodeficiency Virus; LJ: Löwenstein-Jensen; MTC: Mycobacterium tuberculosis complex; NCBI: National Centre for Biotechnology Information; NTM: Non-tuberculous Mycobacteria; PCR: Polymerase Chain reaction; TB: Tuberculosis; WHO: World Health Organization; ZN: Ziehl Neelsen 


\section{Acknowledgements}

The Authors wish to thank JICA and staff at virology laboratory school of veterinary, UNZA and Tropical Diseases Research Centre TB reference laboratory for the assistance rendered in laboratory work.

\section{Funding}

This work was supported by funds from NORAD /NORHED through the CAPAZOMANINTECO project (Grant No. 001/3467/2013).

\section{Availability of data and materials}

The data sets and materials used in the analysis of this current study are readily available from the corresponding author and can be accessed upon reasonable request.

\section{Authors' contributions}

NM, Collected samples, analyzed the data and drafted the manuscript, MM, analyzed the data and supervised data collection, AM, Helped in data analysis, JBM, analyzed and revised the manuscript, SM, conceptualized the study, drafted the manuscript and supervised laboratory work. All authors read and approved the final version of the manuscript.

\section{Ethics approval and consent to participate}

Ethical clearance to conduct the study was sought and granted from "Excellence in Research Ethics and Science" (Ref. No. 2015-Aug-002). Permission to conduct the study in Namwala District was obtained from the National Health Research Authority, Ministry of Health. The study did not pose any risk to the participants and confidentiality was maintained. Written consent was sought from the participants and the objective of the study was properly explained to the participants.

\section{Consent for publication}

Not applicable

\section{Competing interests}

The authors declare that they have no competing interests.

\section{Publisher's Note}

Springer Nature remains neutral with regard to jurisdictional claims in published maps and institutional affiliations.

\section{Author details}

'Department of Biomedical Sciences, Tropical Diseases Research Center, P.O.Box 71769, Ndola, Zambia. ${ }^{2}$ Department of Disease Control, School of Veterinary Medicine, University of Zambia, P.O. Box 32379, 10101 Lusaka, Zambia. ${ }^{3}$ University of Edinburgh, Roslin Institute, Easter Bush, Midlothian, Scotland EH259RG, UK. ${ }^{4}$ Health Promotions Unit, Institute of Economic and Social Research, University of Zambia, P.O. Box 30900, Lusaka, Zambia.

\section{Received: 25 September 2017 Accepted: 25 December 2017}

\section{Published online: 08 January 2018}

\section{References}

1. Ahmed I, Jabeen K, Hasan R. Identification of non-tuberculous mycobacteria isolated from clinical specimens at a tertiary care hospital: a cross-sectional study. BMC Infect Dis. 2013;13:493.

2. Akbar A, Derakhshaninezhad Z, Mirsaeidi M. Nontuberculous mycobacteria in Middle East : current situation and future challenges. Int I Mycobacteriol. 2015;12:1-11.

3. Asiimwe BB, Bagyenzi GB, Ssengooba W, Mumbowa F, Wajja A, Mayanja-kiiza H, Wbudeya E, Kallenius G, Joloba M. Species and genotypic diversity of non-tuberculous mycobacteria isolated from children investigated for pulmonary tuberculosis in rural Uganda. BMC Infect Dis. 2013;13:88.

4. Barber TW, Craven DE, Farber HW. Mycobacterium Gordonae: a possible opportunistic respiratory tract pathogen in patients with advanced human immunodeficiency virus, type 1 infection. Chest. 1991;100:716-20.

5. Buijtels PCAM, Petit LCP, Verbrugh HA, Belkum AV, Soolingen DV. Isolation of Nontuberculous Mycobacteria in Zambia: eight case reports. J Clin Microbiol. 2005;43:6020-6.

6. Buijtels PCAM, Sande ABM, Petit LCP, Verbrugh HA, Belkum AV, Soolingen DV. Nontuberculous Mycobacteria, Zambia. Emerg Infect Dis. 2009;15:242-9.
7. Chanda-kapata P, Kapata N, Klinkenberg E, Mulenga L, Tembo M, Katemangwe P, Sunkutu V, Mwaba P, Grobusch M. Non-tuberculous mycobacteria ( NTM ) in Zambia: prevalence, clinical, radiological and microbiological characteristics. BMC Infect Dis. 2015;15:1264-6.

8. Cloud L, Meyer J, Pounder I, Jost C, Sweeney A, Carroll C, Woods G. Mycobacterium arupense sp. nov., a non-chromogenic bacterium isolated from clinical specimens. Int J Syst Evol Microbiol. 2006;56:1413-8.

9. Covert TC, Rodgers RK, Reyes AL, Stema GN. Occurrence of Nontuberculous Mycobacteria in environmental samples. Appl Environ Microbiol. 1999;65(6):2492-6.

10. Edirisinghe EAR. Occurrence of nontuberculous mycobacteria in aquatic sources of Sri Lanka. Int J Mycobacteriol. 2014;3(4):242-6.

11. Falkinham JO. Epidemiology of infection by Nontuberculous Mycobacteria. Clin Microbiol Rev. 1996;9:177-215.

12. Falkinham JO, Norton DC, LeChevallier MW. Factors influencing numbers of Mycobacterium Avium, mycobacterium intracellulare, and other Mycobacteria in drinking water distribution systems. Appl Environ Microbiol. 2001;67:1225-31.

13. Falkinham JO. Surrounded by mycobacteria : nontuberculous mycobacteria in the human environment. J Appl Microbiol. 2009;107:356-67.

14. Hamid ME, Roth A, Landt O, Kroppenstedt M, Goodfellow M, Mauch H. Differentiation between Mycobacterium farcinogenes and Mycobacterium senegalense strains based on 165-23S ribosomal DNA internal transcribed spacer sequences. J Clin Microbiol. 2002;40:707-11.

15. Hongbao M, Young J, Shen C. RNA, DNA and protein isolation using TRI Zol reagent. Nat Sci. 2008;6(3):66-75.

16. Jarzembowski A, Young MB. Nontuberculous Mycobacterial infections. Arch Pathol Lab Med. 2008;132:1333-41.

17. Kankya C, Muwonge A, Djønne B, Munyeme M, Opuda-Asibo J, Skjerve E, Oloya J, Johansen TB. Isolation of non-tuberculous mycobacteria from pastoral ecosystems of Uganda: public health significance. BMC Public Health. 2011;11:320.

18. Katale BZ, Mbugi VE, Botha L, Keyyus J, Kendall S, Hazel D, Michel A, Kazwala R, Rweyemamu M, Helden P, Matee M. Species diversity of non-tuberculous mycobacteria isolated from humans, livestock and wildlife in the Serengeti ecosystem. BMC Infect Dis. 2014;14:616.

19. Konuk M, Korcan E, Dulgerbaki S, Altindis M. Isolation and identification of Mycobacteria from raw milk samples in Afyonkarahisar district of Turkey. Int J Food Microbiol. 2007;115:343-7.

20. LeDantec C, Duguet J, Mentiel A, Dumoutier D, Vincent V. Occurrence of Mycobacteria in water treatment lines and in water distribution systems. Appl Environ Microbiol. 2002:68:5318-25.

21. Malama S, Munyeme M, Mwanza S, Muma JB. Isolation and characterization of non tuberculous mycobacteria from humans and animals in Namwala District of Zambia. BMC Res Notes. 2014;7:622.

22. Malama S, Muma J, Munyeme M, Mbulo G, Muwonge A, Shamputa IC, Djonne B, Godfroid J, Johansen TB. Isolation and Molecular Characterization of Mycobacterium tuberculosis from Humans and Cattle in Namwala District, Zambia. EcoHealth journal (International Association for Ecology and Health). 2014. doi:https://doi.org/10.1007/s10393-014-0940-0.

23. Munyeme M, Muma JB, Samui KL, Skjerve E, Nambota AM, Phiri IG, Rigouts $\mathrm{L}$, Tryland M. Prevalence of bovine tuberculosis and animal level risk factors for indigenous cattle under different grazing strategies in the livestock/ wildlife interface areas of Zambia. Tropl Anim Health Prod. 2009;41:345-52.

24. Munyeme M, Muma JB, Siamudaala VM, Skjerve E, Munang'andu HM, Tryland M. Tuberculosis in Kafue lechwe antelopes (Kobus Leche Kafuensis) of the Kafue Basin in Zambia. Prev Vet Med. 2010;95:305-8.

25. Muwonge A, Kankya C, Johansen TB, Djonne B, Godfroid J, Biffa D, Edvardsen $V$, Skjerve E. Non-tuberculous mycobacteria isolated from slaughter pigs in Mubende district, Uganda. BMC Vet Res. 2012;8:52.

26. Masaki T, Ohkusu K, Hata H, Fujiwara N, Lihara H, Yama-Noda M, Nhung PH, Hayashi A, Kawamura Y, Ezaki T. Mycobacterium kumamotonense sp. Nov. recovered from clinical specimen and the first isolation report of Mycobacterium arupense in Japan : novel slowly growing, Nonchromogenic clinical isolates related to Mycobacterium terrae Complex, Microbiolofy. Immunology. 2006;50:889-97.

27. Neonakis I, Gitti Z, Kontos F, Spandidos D. Mycobacterium aruprnse pulmonary infection: antibiotic resistance and restriction fragment length polymorphism analysis. Indian J Med Microbiol. 2008;28:173-6.

28. Panagiotou M, Papaioannai Al, Kostitas K, Paraskeya M, Velentza E, Kanellopoulou M, Filaditaki. The Epidemiology of Pulmonary Nontuberculous Mycobacteria: Data from a General Hospital in Athens, Greece, 2007-2013, Pulmonary Medicine. 2014;2014:1-9. 
29. Petroff SA. A new and rapid Method for the Isolation and Cultivation of Tubercle Bacilli Directly From the Sputum and Feces. J Exp Med. 1915;21:38-42.

30. Phiri AM. Common conditions leading to cattle carcass and offal condemnations at 3 abattoirs in the Western Province of Zambia and their zoonotic implications to consumers. J S Afr Vet Assoc. 2006;77:28-32.

31. Primm TP, et al. Health impacts of environmental Mycobacteria. Clin Microbiol Rev. 2004;17(1):98-106.

32. Puthalapanttu S, Metersky M. Mycobacterium Nebraskense as a cause of nudular pulmonary disease. Conn Med. 2011;75:527-9.

33. Ricketts W, Shaughnessy T, Van Ingen J. Human-to-human transmission of Mycobacterium kansasii or victims of a shared source. Eur Respir J. 2014;44: 1085-7.

34. Roth A, Reischl U, Streubel A, Naumann L, Kroppenstedt RM, Habicht M. Novel diagnostic algorithm for identification of mycobacteria using genusspecific amplification of the 16S-23S rRNA gene spacer and restriction endonucleases. J Clin Microbiol. 2000;38:1094-104.

35. Schulze-Robbecke R, Janning B, Fischeder R. Occurrence of mycobacteria in biofilm samples. Tuber Lung Dis. 1992;73(3):141-4.

36. Slany M, Svobodora J, Ettlova A, Slana I, Mrlik V, Pavlik I. Mycobacterium arupense among the isolates of non-tuberculous mycobacteria from human, animal and environmental samples. Vet Med. 2010;55:369-76.

37. Thomson RM, Tolson C, Carter R, Couter C, Huygens F, Hargreavens. Isolation of nontuberculous mycobacteria (NTM) from household water and shower aerosols in patients with pulmonary disease caused by NTM. J Clin Microbiol. 2013:51:3006-11.

38. Thomson RM, Tolson C, Carter R, Couter C, Huygens F, Hargreavens. Factors associated with the isolation of Nontuberculous mycobacteria ( NTM ) from a large municipal water system in Brisbane, Australia. BMC Microbiol. 2013;13:89.

39. Todorova TT, Kaludova V, Tsankova G, Ermenlieva N. A pulmonary infection caused by Mycobacterium peregrinum - a case report. J IMAB. 2015;21:1000-2.

40. Tsai T, Lai C, Tsai I, Chang C, Hsiao P. Tenosynovitis caused by Mycobacterium arupense in a patient with diabetes mellitus. Clin Infect Dis. 2008:47:860-1.

41. Velayati AA, Farnia P, Mozafari M, Malekshahian D, Seif S, Rahideh S, Mirsaeidi M. Molecular epidemiology of nontuberculous mycobacteria isolates from clinical and environmental sources of a metropolitan city. PLoS One. 2014:9:e114428.

42. Velayati AA, Farnia P, Mozatari M, Mehdi M. Nontuberculous Mycobacteria isolation from clinical and environmental samples in Iran: twenty years of surveillance. Biomed Res Int. 2015;2015:1-10.

43. Weinberger M, Berg SL, Feuerstein IM, Pizzo PA, Witebsky FG. Disseminated infection with Mycobacterium Gordonae: report of a case and critical review of the literature. Clin Infect Dis. 1992;14:1229-39.

44. Wu J, Zhang $Y$, Li J, Lin S, Wang L, Jiand $Y$, Pan $Q$, Shen X. Increase in Nontuberculous Mycobacteria isolated in shanghai, China : results from a population-based study. PLoS One. 2014;9:e109736.

\section{Submit your next manuscript to BioMed Central and we will help you at every step:}

- We accept pre-submission inquiries

- Our selector tool helps you to find the most relevant journal

- We provide round the clock customer support

- Convenient online submission

- Thorough peer review

- Inclusion in PubMed and all major indexing services

- Maximum visibility for your research

Submit your manuscript at www.biomedcentral.com/submit 